\title{
BOOST: a phase 3 trial of sorafenib vs. best supportive care in first line treatment of hepatocellular carcinoma in patients with deteriorated liver function
}

\author{
Gennaro Daniele ${ }^{1, a,{ }^{*}}$, Clorinda Schettino ${ }^{1,{ }^{*}}$, Laura Arenare ${ }^{1}$, Domenico Bilancia ${ }^{2}$, Fabio Farinati ${ }^{3}$, Piera \\ Federico $^{4, c}$, Stefano Tamberi ${ }^{5}$, Gino Crivellari ${ }^{6}$, Sandro Barni ${ }^{7}$, Raffaella Tortora ${ }^{8}$, Francesco Izzo ${ }^{9}$, Antonio \\ Frassoldati $^{10}$, Luigi Cavanna ${ }^{11}$, Claudia Mucciarini ${ }^{12}$, Luigi Bolondi ${ }^{13}$, Angelo Dinota ${ }^{2}$, Filippo Pelizzaro ${ }^{3}$, \\ Maria Carmela Piccirillo ${ }^{1}$, Piera Gargiulo ${ }^{1}$, Massimo Di Maio ${ }^{1, b}$, Ciro Gallo ${ }^{14}$, Francesco Perrone ${ }^{1, \#, ~ B r u n o ~}$ \\ Daniele ${ }^{4, c, \#}$
}

'Unità Sperimentazioni Cliniche, Istituto Nazionale Tumori, IRCCS, Fondazione G. Pascale, Napoli 80131, Italy.

${ }^{2}$ Oncologia Medica, Azienda Ospedaliera S. Carlo, Potenza 80131, Italy.

${ }^{3}$ Unità di Gastroenterologia, Dipartimento di Scienze Chirurgiche, Oncologiche e Gastroenterologiche, Università di Padova,

Padova 35121, Italy.

${ }^{4}$ Oncologia, Azienda Ospedaliera G. Rummo, Benevento 82100, Italy.

${ }^{5}$ Oncologia Medica, Ospedale Civile degli Infermi, Faenza (RA) 48018, Italy.

${ }^{6}$ Oncologia Medica 1, Istituto Oncologico Veneto, Dipartimento di Oncologia Clinica e Sperimentale, Padova 35128, Italy.

${ }^{7}$ Oncologia Medica, Azienda Ospedaliera Treviglio - Caravaggio, Treviglio (BG) 24047, Italy.

${ }^{8}$ UOC Epatologia, Dipartimento dei Trapianti, AORN A. Cardarelli, Napoli 80131, Italy.

${ }^{9}$ Chirurgia Epato-biliare, Istituto Nazionale Tumori, IRCCS Fondazione G. Pascale, Napoli 80131, Italy.

${ }^{10}$ Oncologia Clinica, Azienda Ospedaliera Universitaria Arcispedale Sant' Anna, Ferrara 44124, Italy.

${ }^{11}$ Oncologia Medica ed Ematologia, USL di Piacenza, Ospedale Guglielmo da Saliceto, Piacenza 29121, Italy.

${ }^{12}$ UOC Medicina Oncologica, Ospedale Ramazzini, Carpi (MO) 41012, Italy.

${ }^{13}$ Medicina Interna, Policlinico S. Orsola-Malpighi, Bologna 40138, Italy.

${ }^{14}$ Statistica Medica, Università della Campania Luigi Vanvitelli, Napoli 81055, Italy.

a(Present addresses) Fondazione Policlinico Universitario A. Gemelli, IRCCS, Univer-sita' Cattolica, Roma, Italy.

' (Present addresses) Dipartimento di Oncologia, Università degli Studi di Torino - Oncologia, A. O. Ordine Mauriziano, Ospedale Umberto I, Torino, Italy.

'(Present addresses) Oncologia, Ospedale del Mare, Napoli, Italy.

${ }^{\star}$ Co-first author; ${ }^{*}$ co last authors.

Correspondence to: Bruno Daniele, MD, Oncologia, Ospedale del Mare, Via Enrico Russo, Napoli 80147, Italy.

E-mail: bruno.daniele@asInapoli1centro.it

How to cite this article: Daniele G, Schettino C, Arenare L, Bilancia D, Farinati F, Federico P, Tamberi S, Crivellari G, Barni S, Tortora R, Izzo F, Frassoldati A, Cavanna L, Mucciarini C, Bolondi L, Dinota A, Pelizzaro F, Piccirillo MC, Gargiulo P, Di Maio M, Gallo C, Perrone F, Daniele B. BOOST: a phase 3 trial of sorafenib vs. best supportive care in first line treatment of hepatocellular carcinoma in patients with deteriorated liver function. Hepatoma Res 2021;7:61. https://dx.doi.org/10.20517/2394-

5079.2021 .58

Received: 21 Apr 2021 First Decision: 31 May 2021 Revised: 30 Jun 2021 Accepted: 20 Jul 2021 First online: 29 Jul 2021 


\begin{abstract}
Aim: Only patients with good liver function \{[Child-Pugh (CP)] A class $\}$ were eligible for trials testing sorafenib as first-line treatment of hepatocellular carcinoma (HCC); nevertheless, the drug was authorized without restrictions based on liver function. Therefore, we planned to test sorafenib efficacy and safety in patients with HCC and deteriorated liver function (CP-B).
\end{abstract}

Methods: This was an open-label, multicenter, randomized phase 3 trial. Patients with HCC, no previous systemic therapy, and CP-B score 7-9 were assigned 1:1 to best supportive care alone (control arm) or with standard dose sorafenib (experimental arm). Overall survival (OS) was the primary endpoint. To detect a 0.70 HR of death, with $80 \%$ power, and two-tailed $\alpha$ error 0.05, 234 events were required. The study closed prematurely because of slow accrual. Descriptive analyses are reported.

Results: From 2012 to 2017, 13 Italian centers randomized 35 patients. In total, 28 deaths were recorded, 12 without and 16 with sorafenib; median OS was 4.9 (95\% Cl: 1.2-5.6) and 3.5 months (95\% Cl: 1.3-5.3), respectively. At least one severe adverse event was reported in 2/15 (13.3\%) without and 9/17 (52.9\%) patients with sorafenib.

Conclusions: This trial failed its planned enrolment goal, showing the difficulty in performing clinical trials with drugs already registered with a label broader than what available evidence supports.

Keywords: Hepatocellular carcinoma, Child-Pugh B class, sorafenib

\title{
INTRODUCTION
}

In 2018, hepatocellular carcinoma (HCC) represented the 6th most common human cancer (over 840,000 new cases) and the fourth most common cause of cancer-related death (780,000 estimated deaths), according to the International Agency for Research on Cancer database ${ }^{[1]}$. Estimated ranking of incidence and mortality in 2019 in the United States suggests that incidence is going to decrease as compared to other types of cancer, but mortality remains significant ${ }^{[2]}$. Prognosis depends on both the tumor characteristics and the liver failure due to concomitant cirrhosis; thus, Child-Pugh score and other markers of liver function are included in several HCC staging systems ${ }^{[3-5]}$.

In patients with advanced untreated HCC, the prognosis is extremely poor, yielding a median survival of 47 months ${ }^{[6]}$.

Sorafenib is an oral multi-kinase inhibitor of vascular endothelial growth factor receptors 1,2 , and 3 , the platelet-derived growth factor receptor, and the RAF pathways ${ }^{[7]}$. In two randomized phase 3 trials (SHARP and Asia-Pacific), it prolonged overall survival and time to progression compared to placebo in patients with advanced HCC who had not received prior systemic therapy ${ }^{[8,9]}$.

In both trials, only patients with good liver function (Child-Pugh A) were eligible and few Child-Pugh B 
cases (20 and 6 patients in SHARP and Asia Pacific, respectively) were enrolled as protocol violations. Nevertheless, the Food and Drug Administration and European Medicines Agency approved sorafenib for the treatment of patients with advanced HCC regardless of liver function. However, the cost-benefit ratio is unknown in patients with highly compromised liver function, and sorafenib is reimbursed only for ChildPugh A patients in several countries (Australia, Belgium, Canada, France, Italy, South Korea, Taiwan, and Switzerland) $)^{[10]}$.

In 2011, we planned the BOOST (B Child-Pugh HCC patients - Optimization Of Sorafenib Treatment) randomized phase 3 trial to assess the efficacy and safety of sorafenib plus best supportive care (BSC) vs. BSC alone in Child-Pugh B advanced HCC patients.

\section{PATIENTS AND METHODS}

\section{Study management and design}

BOOST (NCT:01405573, EudraCT number: 2009-013870-42) was an open-label, multicenter, randomized phase 3 trial promoted by the Istituto Nazionale per lo Studio e la Cura dei Tumori - IRCCS Fondazione G. Pascale, Napoli, Italy. The study was supported by the Italian Drug Agency (AIFA) in May 2011 (code FARM84SA2X) for trial coordination activities. A request to the pharmaceutical company for gratuitous drug supply was unsuccessful. Therefore, only 15 centers, willing to pay for the experimental drug, accepted to participate, but the enrolment was quite null. In August 2014, AIFA agreed on further funding to buy the experimental drug. The number of centers willing to participate increased to 36, but only 13 actually enrolled at least one patient. Figure 1 summarizes time dynamics of the trial.

Patients were randomly assigned, in a 1:1 ratio, to receive BSC alone (control arm) or combined with sorafenib (experimental arm) until disease progression, occurrence of unacceptable toxicity, or progression of the underlying cirrhosis.

Participants provided written informed consent before any study procedures.

\section{Eligibility criteria}

Eligible patients had liver function classified as Child-Pugh B and advanced HCC (according to the American Association for the Study of Liver Diseases and the European Association for the Study of the Liver criteria $)^{[11]}$, were not eligible for loco-regional treatment, and had not received previous systemic treatment. Other inclusion criteria were age $\geq 18$ years, Eastern Cooperative Oncology Group (ECOG) performance status (PS) score 0-2, life expectancy longer than 2 months, adequate hematologic (platelet count $\geq 60.0 \times 10^{9} / \mathrm{L}$; hemoglobin $>9 \mathrm{~g} / \mathrm{dL}$ ) and renal function (serum creatinine $<1.5$ times the upper limit of normal range), and signed informed consent.

Key exclusion criteria were the presence of any unstable systemic disease or medical contraindication to sorafenib and any grade encephalopathy or gastrointestinal hemorrhage within 30 days before the randomization.

\section{Treatment and study procedures}

Sorafenib, at the starting dose of $800 \mathrm{mg}$ (two $200 \mathrm{mg}$ tablets every $12 \mathrm{~h}$ ), was assumed orally on a continuous daily basis; for convenience, the treatment period was divided into 4-week cycles. Based on the occurrence and the severity of side effects (diarrhea, skin toxicity, hematologic, or other non-hematologic adverse events) or Child-Pugh deterioration, sorafenib was allowed to be stepwise reduced to $400 \mathrm{mg}$ daily (200 mg bid) or $400 \mathrm{mg}$ every other day. After such reductions, in case of persistent toxicity, treatment had 


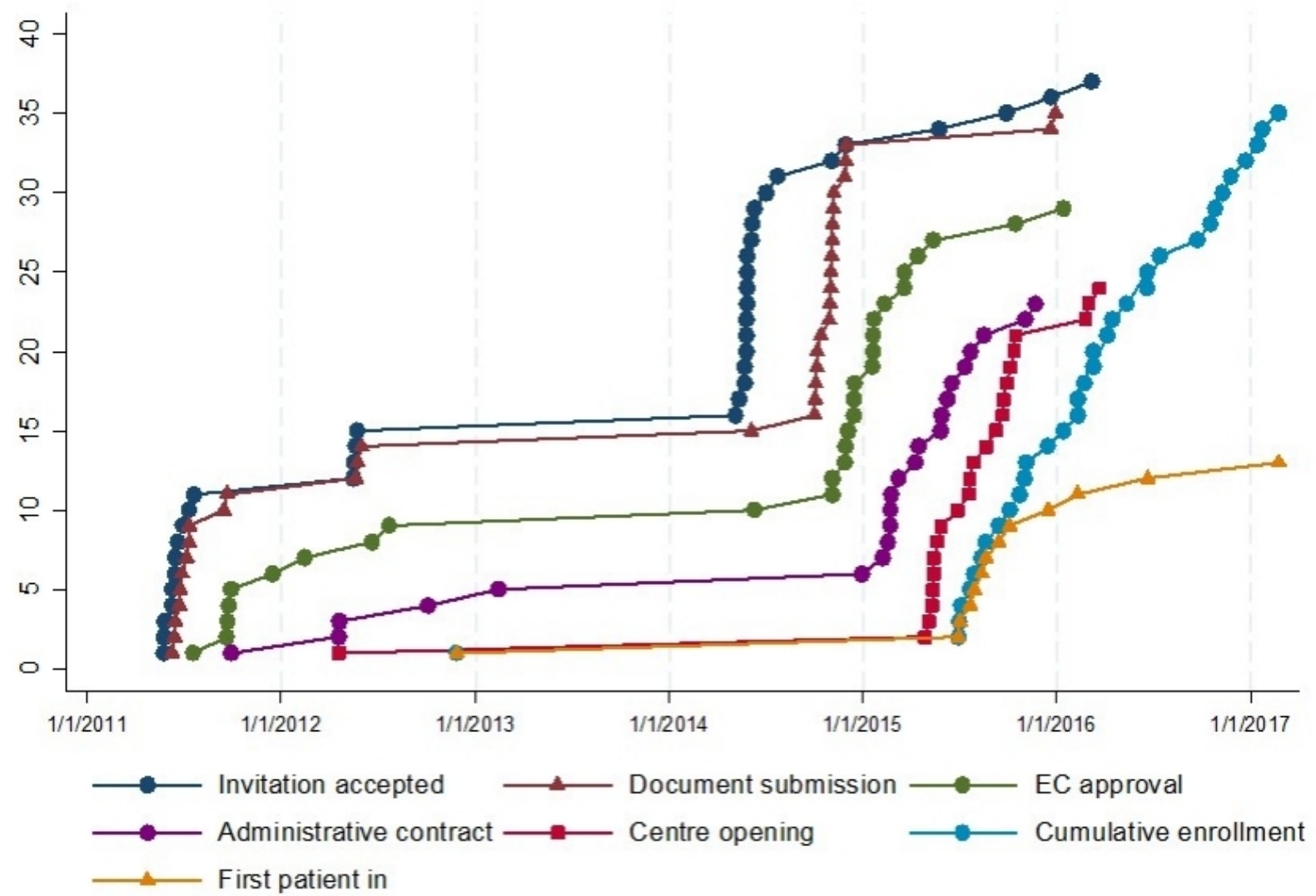

Figure 1. Time dynamics of trial conduction.

to be definitively stopped.

Randomization was performed centrally, at the Clinical Trials Unit of the Istituto Nazionale per lo Studio e la Cura dei Tumori - IRCCS Fondazione G. Pascale, Napoli, Italy, through a web-based minimization procedure, with Child-Pugh score (7 vs. 8 vs. 9), Cancer Liver Italian Program (CLIP) score (1 vs. 2-3 vs. 4$5)$, and age ( $<70 v s . \geq 70)$ as strata.

\section{Endpoints}

The primary end point of the study was overall survival (OS), defined as the time between the date of randomization and the date of death from any cause.

Secondary endpoints included toxicity, quality of life (QoL), and progression-free survival (PFS).

Toxicity was evaluated in both arms at baseline and every four weeks until disease progression. Adverse events were coded according to Common Terminology Criteria for Adverse Events version 4.03. Toxicity was described for each item as the worst grade suffered by the patient at any time during the treatment.

QoL was measured with the European Organization for Research and Treatment of Cancer (EORTC) QLQC30 questionnaire and the HCC specific module (EORTC QLQ-HCC 18$)^{[12,13]}$ at baseline and every four weeks, until 24 weeks, in both treatment arms. 
Baseline tumor measurement, with abdominal and pelvic computed tomography scan or magnetic resonance, was planned for all patients, before the randomization, and in the sorafenib arm only, every eight weeks during treatment, until disease progression. PFS, defined as the time between the date of randomization and the date of disease progression [according to the modified Response Evaluation Criteria in Solid Tumors (RECIST 1.1) $]^{[14]}$ or death, whichever occurred first, was described only in the sorafenib arm. For this purpose, patients who did not progress or die were censored at the date of the last available information on vital status.

\section{Statistical analysis}

Sample size calculation was based on an expected median survival of 4.5 months in the control arm and an auspicated 6.5-month median survival in the sorafenib arm, corresponding to a hazard ratio (HR) of death of 0.70 . With a two-tailed alpha error of 0.05 , and $80 \%$ power, 234 events were required, and it was planned to enroll 320 patients in two years (EAST 3.1, Cytel Software, Cambridge, MA, USA).

In March 2017, the study was stopped due to slow enrolment, with 35 patients randomized. Due to low study power, only descriptive analyses are reported.

Median follow-up was calculated according to the Schemper's reverse Kaplan-Meier technique ${ }^{[15]}$.

Survival curves were drawn with the Kaplan-Meier product-limit method, and the HR of death was calculated with the Cox proportional hazard model.

Toxicity was described for all the patients with available toxicity information. Side-effects were grouped either as any grade (Grade $\geq 1$ ) or as severe (Grade $\geq 3$ ).

Only baseline QoL was descripted due to the high number of missing QoL data through the follow up.

Analyses were performed using STATA MP 14.1 (StataCorp LP, College Station, TX, USA).

\section{RESULTS}

\section{Patients' characteristics}

From 27 November 2012 to 2 February 2017, 13 Italian centers enrolled 35 patients (18 were assigned to the experimental and 17 to the control arm). One patient in the control arm withdrew consent immediately after randomization [Figure 2].

Notwithstanding the small number of patients, the baseline characteristics were well balanced between study arms [Table 1]. Median age was 64.8 years [interquartile range (IQR): 59.2-71.3 years]; 85.3\% of patients were male, and $73.0 \%$ had an ECOG PS 0-1. Child-Pugh scores 7, 8, and 9 were similarly represented in the study population; only few patients (8.8\%) were in the lowest CLIP score category, the majority of the patients (64.7\%) being in the CLIP 2-3 category. Chronic hepatitis C virus infection was the predominant cause of liver disease (67.7\% of the cases), followed by alcohol consumption, and HBV infection. Overall, 16 patients $(47.1 \%)$ had been previously treated with locoregional therapy.

\section{Treatment compliance}

Data on compliance to sorafenib are missing for one patient. The median duration of sorafenib treatment was 28 days (IQR: 20-60 days). The profile of dosing per day across the first two cycles is reported in Figure 3. There were three violations regarding the sorafenib initial dose, with a starting dose of $400 \mathrm{mg}$ 
Table 1. Characteristics of patients according to study arm

\begin{tabular}{|c|c|c|c|c|}
\hline Age & \multicolumn{2}{|r|}{$\operatorname{BSC}^{\dagger}(n=16)$} & \multicolumn{2}{|r|}{$\begin{array}{c}\mathrm{BSC}^{\dagger}+\text { Sorafenib } \\
(n=18)\end{array}$} \\
\hline median (IQR) & 65.6 & $(61.0 ; 70.5)$ & 64.4 & $(53.5 ; 72.9)$ \\
\hline$<70$ & 63.2 & $(59.7 ; 67.0)$ & 60.2 & $(49.8 ; 64.4)$ \\
\hline 70 & 75.4 & $(73.1 ; 77.6)$ & 75.9 & $(72.9 ; 76.8)$ \\
\hline \multicolumn{5}{|l|}{ Gender } \\
\hline Male & 13 & $(81.3 \%)$ & 16 & $(88.9 \%)$ \\
\hline Female & 3 & $(18.8 \%)$ & 2 & $(11.1 \%)$ \\
\hline \multicolumn{5}{|l|}{ Child-Pugh score } \\
\hline 7 & 3 & $(18.8 \%)$ & 6 & $(33.3 \%)$ \\
\hline 8 & 7 & $(43.8 \%)$ & 7 & $(38.9 \%)$ \\
\hline 9 & 6 & $(37.5 \%)$ & 5 & $(27.8 \%)$ \\
\hline \multicolumn{5}{|l|}{ CLIP' score } \\
\hline 1 & 1 & $(6.3 \%)$ & 2 & $(11.1 \%)$ \\
\hline $2-3$ & 11 & $(68.8 \%)$ & 11 & $(61.1 \%)$ \\
\hline $4-5$ & 4 & $(25.0 \%)$ & 5 & $(27.8 \%)$ \\
\hline \multicolumn{5}{|c|}{$\mathrm{ECOG}^{\S}$ performance status } \\
\hline $0-1$ & 11 & $(68.8 \%)$ & 14 & $(77.8 \%)$ \\
\hline 2 & 5 & $(31.3 \%)$ & 4 & $(22.2 \%)$ \\
\hline Aetiology' & 3 & $(18.8 \%)$ & 3 & $(16.7 \%)$ \\
\hline HBV infection & 3 & $(18.8 \%)$ & 3 & $(16.7 \%)$ \\
\hline HBV DNA positive & 1 & $(6.3 \%)$ & 2 & $(11.1 \%)$ \\
\hline HCV infection & 12 & $(75.0 \%)$ & 11 & $(61.1 \%)$ \\
\hline HCV RNA positive & 4 & $(25.0 \%)$ & 2 & $(11.1 \%)$ \\
\hline Alcohol & 6 & $(37.5 \%)$ & 8 & $(44.4 \%)$ \\
\hline \multicolumn{5}{|l|}{ Esophageal varices } \\
\hline No & 6 & $(37.5 \%)$ & 7 & $(38.9 \%)$ \\
\hline Present & 8 & $(50.0 \%)$ & 10 & $(55.6 \%)$ \\
\hline Previous & 2 & $(12.5 \%)$ & 1 & $(5.6 \%)$ \\
\hline \multicolumn{5}{|l|}{ Ascites } \\
\hline No & 5 & $(31.3 \%)$ & 6 & (33.3\%) \\
\hline Mild & 9 & (56.3\%) & 12 & $(66.7 \%)$ \\
\hline Moderate & 2 & $(12.5 \%)$ & 0 & $(0.0 \%)$ \\
\hline \multicolumn{5}{|l|}{ Encephalopathy } \\
\hline Absent & 15 & (93.8\%) & 18 & $(100.0 \%)$ \\
\hline Mild to moderate & 1 & $(6.3 \%)$ & 0 & $(0.0 \%)$ \\
\hline Severe & 0 & $(0.0 \%)$ & 0 & $(0.0 \%)$ \\
\hline \multicolumn{5}{|l|}{ Serum bilirubin } \\
\hline$<2.0 \mathrm{mg} / \mathrm{dL}$ & 6 & $(37.5 \%)$ & 7 & $(38.9 \%)$ \\
\hline $2-3 \mathrm{mg} / \mathrm{dL}$ & 4 & (25.0\%) & 7 & $(38.9 \%)$ \\
\hline$>3.0 \mathrm{mg} / \mathrm{dL}$ & 6 & $(37.5 \%)$ & 4 & $(22.2 \%)$ \\
\hline \multicolumn{5}{|l|}{ Serum albumin } \\
\hline$>3.5 \mathrm{~g} / \mathrm{dL}$ & 1 & $(6.3 \%)$ & 1 & $(5.6 \%)$ \\
\hline $2.8-3.5 \mathrm{~g} / \mathrm{dL}$ & 10 & $(62.5 \%)$ & 10 & $(55.6 \%)$ \\
\hline$<2.8 \mathrm{~g} / \mathrm{dL}$ & 5 & $(31.3 \%)$ & 7 & $(38.9 \%)$ \\
\hline \multicolumn{5}{|l|}{ Prothrombin time } \\
\hline INR $<1.7$ & 15 & $(93.8 \%)$ & 17 & $(94.4 \%)$ \\
\hline
\end{tabular}


INR 1.7-2.3

INR $>2.3$

Morphology of hepatocellular carcinoma

Uninodular and extension $\leq 50 \%$

Multinodular and extension $\leq 50 \%$

Massive or extension $>50 \%$

Alpha-fetoprotein

$<400 \mu \mathrm{g} / \mathrm{L}$

$\geq 400 \mu \mathrm{g} / \mathrm{L}$

Portal vein thrombosis

No

Yes

Previous therapy $\Delta$

Surgical resection

Percutaneous ethanol injection

Radiofrequency ablation

TAE/TACE ${ }^{\mathbb{P}}$

None

$\begin{array}{llll}1 & (6.3 \%) & 0 & (0.0 \%) \\ 0 & (0.0 \%) & 1 & (5.6 \%) \\ 2 & (12.5 \%) & 2 & (11.1 \%) \\ 13 & (81.3 \%) & 13 & (72.2 \%) \\ 1 & (6.3 \%) & 3 & (16.7 \%) \\ 10 & (62.5 \%) & 12 & (75.0 \%) \\ 6 & (37.5 \%) & 6 & (37.5 \%) \\ 7 & & & \\ 9 & (43.8 \%) & 8 & (44.4 \%) \\ & (56.3 \%) & 10 & (62.5 \%)\end{array}$

$\begin{array}{llll}2 & (12.5 \%) & 0 & (0.0 \%) \\ 2 & (12.5 \%) & 5 & (31.3 \%) \\ 4 & (25.0 \%) & 6 & (37.5 \%) \\ 8 & (50.0 \%) & 4 & (25.0 \%) \\ 8 & (50.0 \%) & 10 & (55.6 \%)\end{array}$

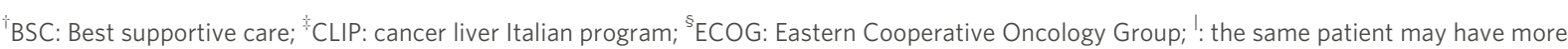
than one aetiologic factors; $\Delta$ : the same patient may have received more than one previous treatment; TAE/TACE : transarterial embolization/transarterial chemoembolization.

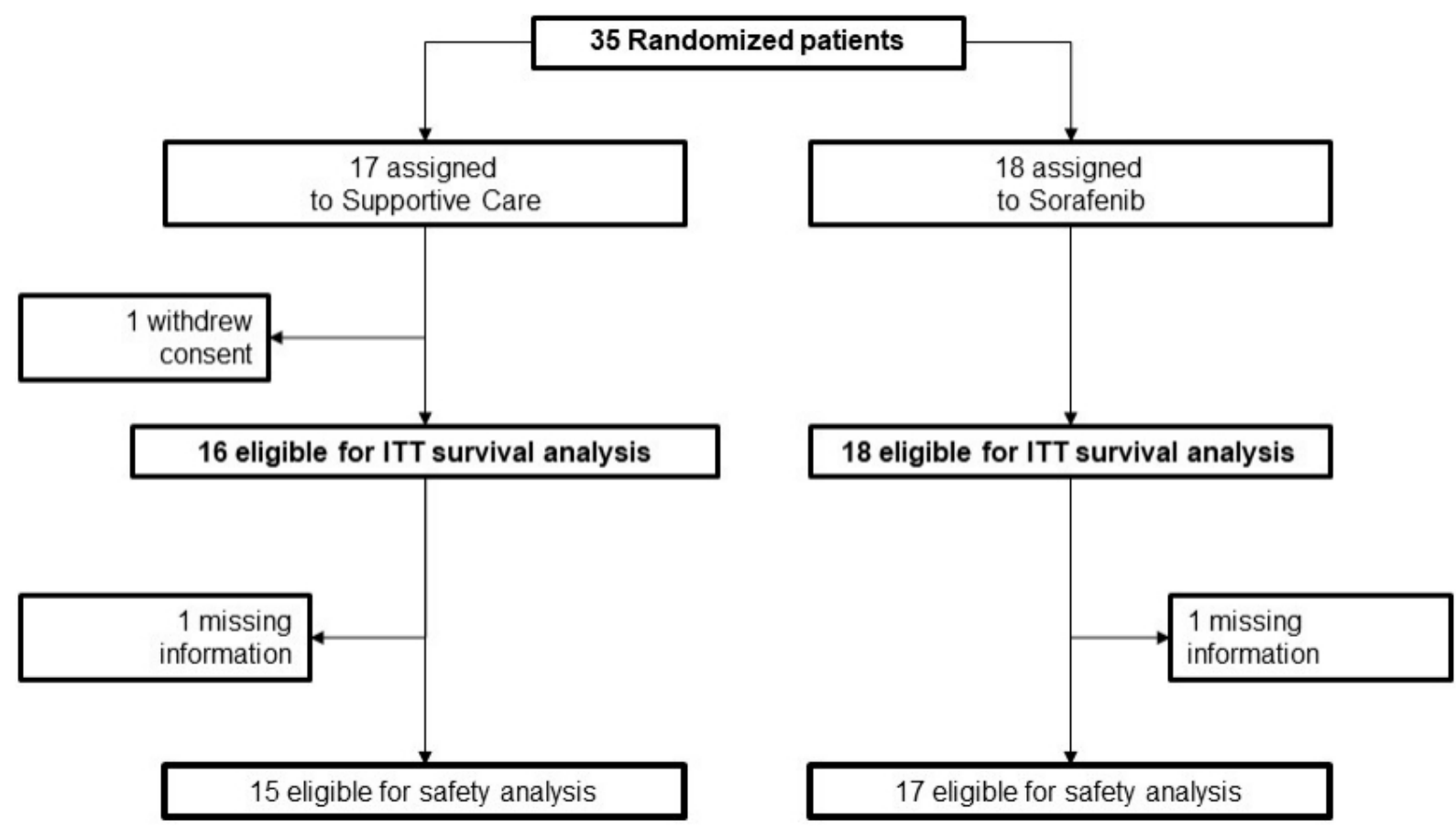

Figure 2. Study flow.

daily rather than $800 \mathrm{mg}$. Four patients required a dose reduction during the treatment. At the time of the analysis, all patients had discontinued sorafenib, 11 of them after one cycle. Reasons for treatment discontinuation were progression of HCC ( 6 cases), toxicity ( 5 cases), patient refusal ( 3 cases, 2 of whom with diarrhea), and worsening from Child-Pugh B to C (2 cases). Adverse events leading to treatment 


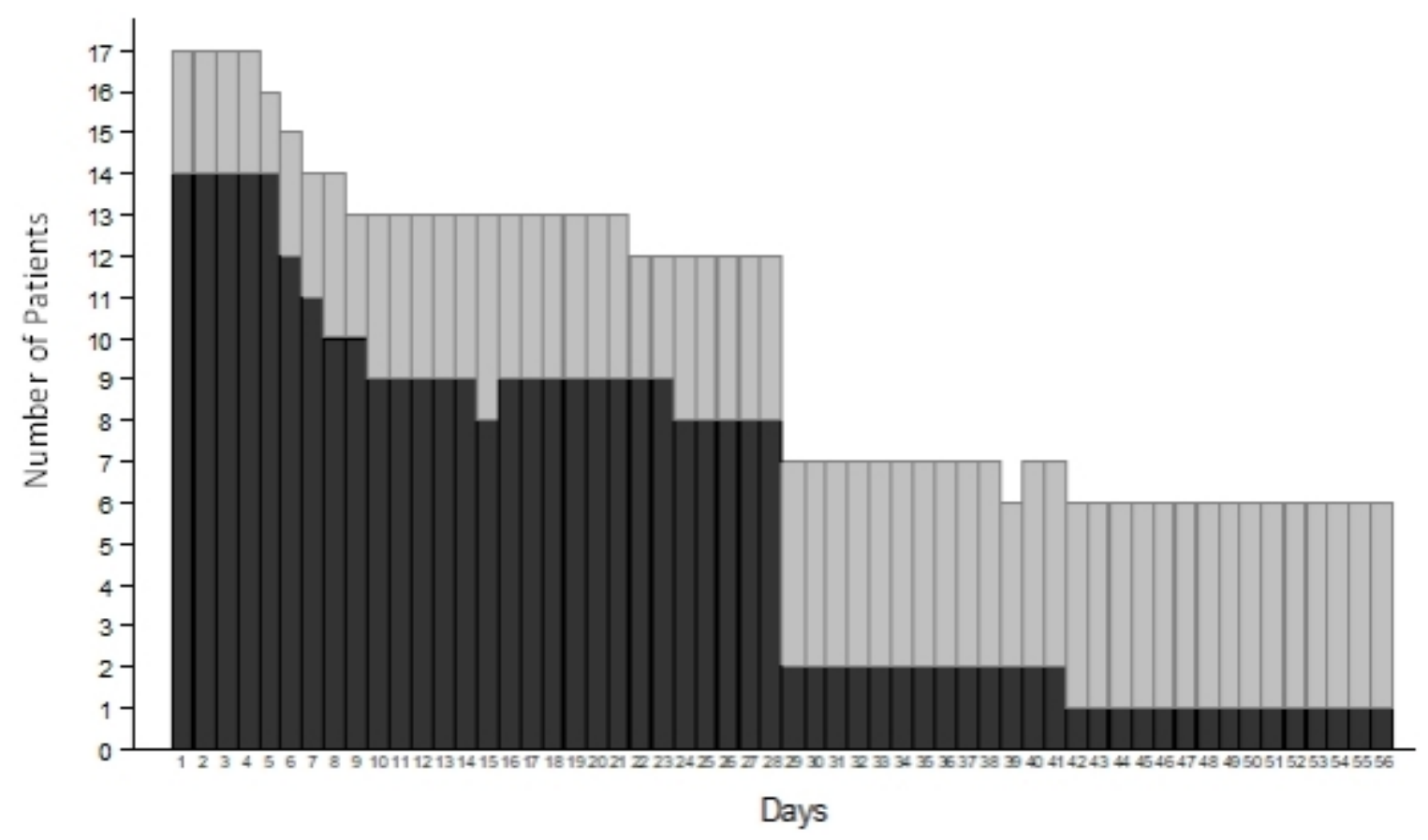

$\square$ Dose $800 \mathrm{mg}$

\section{Dose $<800 \mathrm{mg}$}

Figure 3. Profile of sorafenib dosing over the first two cycles.

discontinuation were bilirubin increase ( 2 cases), fatigue ( 2 cases), and abdominal pain, nausea, vomiting, skin rash, and anemia (1 case each); multiple adverse events were associated in two patients.

\section{Efficacy}

With the database locked on 18 January 2019, 28 deaths were recorded, 12 in the control arm and 16 in the experimental arm: median OS was 4.9 (95\%CI: 1.2-5.6) and 3.5 (95\%CI: 1.3-5.3) months in the two arms, respectively [Figure $4 \mathrm{~A}$ ]. In the experimental arm, 6 patients progressed and 13 died without clinical or radiologic progression; median PFS was 2.4 (95\%CI: 1.2-3.0) months [Figure $4 \mathrm{~B}$ ].

\section{Safety}

Safety analysis included 15 patients in the control and 17 in the experimental arm. There were no toxic deaths. At least one severe adverse event was reported in 2/15 (13.3\%) patients in the control arm (always Grade 3), and in $9 / 17$ (52.9\%) in the sorafenib arm (always Grade 3, but with one case of Grade 4 AST increase). In addition, at least one adverse event (any grade) was reported in 6/15 (40.0\%) patients in the control arm and in 15/17 (88.2\%) in the sorafenib. Table 2 summarizes side-effects by grade and treatment arm.

\section{QoL}

Baseline QoL questionnaires were completed by 9 of 16 patients $(56.2 \%)$ in the control arm and 12 of 18 patients (67\%) in the experimental one. The mean global QoL score (Items 29 and 30 of the EORTC-C30) was 53.7 in the control arm and 62.5 in the experimental one. Thereafter, compliance dramatically decreased (with 3 and 4 questionnaires filled in after one cycle, respectively) and prevented any description 
Table 2. Any grade and severe toxicity according to CTCAE categories and subcategories, by treatment arm

\begin{tabular}{|c|c|c|c|c|c|c|c|c|}
\hline \multirow{3}{*}{ Toxicity } & \multicolumn{4}{|c|}{ Any grade $(>0)$} & \multicolumn{4}{|c|}{ Severe $(\geq 3)$} \\
\hline & \multicolumn{2}{|c|}{$\operatorname{BSC}^{\dagger}(n=15)$} & \multicolumn{2}{|c|}{ BSC $^{\dagger}+$ Sorafenib $(n=17)$} & \multicolumn{2}{|c|}{$\mathrm{BSC}^{\dagger}(n=15)$} & \multicolumn{2}{|c|}{ BSC $^{\dagger}+$ Sorafenib $(n=17)$} \\
\hline & 6 & $(40.0 \%)$ & 15 & $(88.2 \%)$ & 2 & $(13.3 \%)$ & 9 & $(52.9 \%)$ \\
\hline \multicolumn{9}{|l|}{ Blood } \\
\hline Anemia & 1 & $(6.7 \%)$ & 3 & $(17.6 \%)$ & 0 & $(0.0 \%)$ & 2 & $(11.8 \%)$ \\
\hline \multicolumn{9}{|l|}{ Cardiac } \\
\hline Atrial fibrillation & 0 & $(0.0 \%)$ & 1 & $(5.9 \%)$ & 0 & $(0.0 \%)$ & 0 & $(0.0 \%)$ \\
\hline \multicolumn{9}{|l|}{ Gastrointestinal } \\
\hline Abdominal distension & 0 & $(0.0 \%)$ & 2 & $(11.8 \%)$ & 0 & $(0.0 \%)$ & 0 & $(0.0 \%)$ \\
\hline Abdominal pain & 0 & $(0.0 \%)$ & 4 & $(23.5 \%)$ & 0 & $(0.0 \%)$ & 1 & $(5.9 \%)$ \\
\hline Ascites & 3 & $(20.0 \%)$ & 5 & $(29.4 \%)$ & 1 & $(6.7 \%)$ & 1 & $(5.9 \%)$ \\
\hline Constipation & 1 & $(6.7 \%)$ & 1 & $(5.9 \%)$ & 1 & $(6.7 \%)$ & 0 & $(0.0 \%)$ \\
\hline Diarrhea & 1 & $(6.7 \%)$ & 4 & $(23.5 \%)$ & 0 & $(0.0 \%)$ & 1 & $(5.9 \%)$ \\
\hline Dry mouth & 0 & $(0.0 \%)$ & 1 & $(5.9 \%)$ & 0 & $(0.0 \%)$ & 0 & $(0.0 \%)$ \\
\hline Mucositis (oral) & 0 & $(0.0 \%)$ & 1 & $(5.9 \%)$ & 0 & $(0.0 \%)$ & 0 & $(0.0 \%)$ \\
\hline Nausea & 1 & $(6.7 \%)$ & 4 & (23.5\%) & 0 & $(0.0 \%)$ & 1 & $(5.9 \%)$ \\
\hline \multicolumn{9}{|l|}{ General disorders } \\
\hline Fatigue & 3 & $(20.0 \%)$ & 6 & $(35.3 \%)$ & 0 & $(0.0 \%)$ & 2 & $(11.8 \%)$ \\
\hline Fever & 1 & $(6.7 \%)$ & 0 & $(0.0 \%)$ & 0 & $(0.0 \%)$ & 0 & $(0.0 \%)$ \\
\hline Other & 1 & $(6.7 \%)$ & 1 & $(5.9 \%)$ & 0 & $(0.0 \%)$ & 0 & $(0.0 \%)$ \\
\hline Pain & 0 & $(0.0 \%)$ & 1 & $(5.9 \%)$ & 0 & $(0.0 \%)$ & 0 & $(0.0 \%)$ \\
\hline \multicolumn{9}{|l|}{ Investigation } \\
\hline Amylase increased & 0 & $(0.0 \%)$ & 1 & $(5.9 \%)$ & 0 & $(0.0 \%)$ & 1 & $(5.9 \%)$ \\
\hline ALP increase & 1 & $(6.7 \%)$ & 4 & $(23.5 \%)$ & 0 & $(0.0 \%)$ & 0 & $(0.0 \%)$ \\
\hline ALT increase & 1 & $(6.7 \%)$ & 4 & $(23.5 \%)$ & 0 & $(0.0 \%)$ & 3 & $(17.6 \%)$ \\
\hline AST increase & 1 & $(6.7 \%)$ & 4 & (23.5\%) & 0 & $(0.0 \%)$ & 1 & $(5.9 \%)$ \\
\hline Bilirubin increased & 2 & $(13.3 \%)$ & 7 & (41.2\%) & 0 & $(0.0 \%)$ & 1 & $(5.9 \%)$ \\
\hline GGT increased & 0 & $(0.0 \%)$ & 2 & $(11.8 \%)$ & 0 & $(0.0 \%)$ & 0 & $(0.0 \%)$ \\
\hline Haptoglobin decreased & 0 & $(0.0 \%)$ & 1 & $(5.9 \%)$ & 0 & $(0.0 \%)$ & 0 & $(0.0 \%)$ \\
\hline Weight gain & 0 & $(0.0 \%)$ & 1 & $(5.9 \%)$ & 0 & $(0.0 \%)$ & 0 & $(0.0 \%)$ \\
\hline \multicolumn{9}{|l|}{ Metabolism } \\
\hline Anorexia & 1 & $(6.7 \%)$ & 5 & $(29.4 \%)$ & 0 & $(0.0 \%)$ & 0 & $(0.0 \%)$ \\
\hline Dehydration & 2 & $(13.3 \%)$ & 1 & $(5.9 \%)$ & 0 & $(0.0 \%)$ & 1 & $(5.9 \%)$ \\
\hline \multicolumn{9}{|l|}{ Nervous system } \\
\hline Encephalopathy & 1 & $(6.7 \%)$ & 1 & $(5.9 \%)$ & 0 & $(0.0 \%)$ & 0 & $(0.0 \%)$ \\
\hline \multicolumn{9}{|l|}{ Psychiatric } \\
\hline Agitation & 0 & $(0.0 \%)$ & 1 & $(5.9 \%)$ & 0 & $(0.0 \%)$ & 0 & $(0.0 \%)$ \\
\hline Depression & 0 & $(0.0 \%)$ & 1 & $(5.9 \%)$ & 0 & $(0.0 \%)$ & 0 & $(0.0 \%)$ \\
\hline Restlessness & 0 & $(0.0 \%)$ & 1 & $(5.9 \%)$ & 0 & $(0.0 \%)$ & 0 & $(0.0 \%)$ \\
\hline \multicolumn{9}{|l|}{ Skin } \\
\hline Erythema & 0 & $(0.0 \%)$ & 1 & $(5.9 \%)$ & 0 & $(0.0 \%)$ & 0 & $(0.0 \%)$ \\
\hline $\mathrm{PPE}^{+}$ & 0 & $(0.0 \%)$ & 3 & $(17.6 \%)$ & 0 & $(0.0 \%)$ & 0 & $(0.0 \%)$ \\
\hline Pruritus & 1 & $(6.7 \%)$ & 1 & $(5.9 \%)$ & 0 & $(0.0 \%)$ & 0 & $(0.0 \%)$ \\
\hline Rash maculo papular & 0 & $(0.0 \%)$ & 2 & $(11.8 \%)$ & 0 & $(0.0 \%)$ & 0 & $(0.0 \%)$ \\
\hline \multicolumn{9}{|l|}{ Vascualar } \\
\hline Hypertension & 0 & $(0.0 \%)$ & 4 & $(23.5 \%)$ & 0 & $(0.0 \%)$ & 0 & $(0.0 \%)$ \\
\hline Hypotension & 0 & $(0.0 \%)$ & 1 & (5.9\%) & 0 & $(0.0 \%)$ & 0 & $(0.0 \%)$ \\
\hline
\end{tabular}

'BSC: Best supportive care; "PPE: palmar-plantar erythrodysesthesia syndrome. 

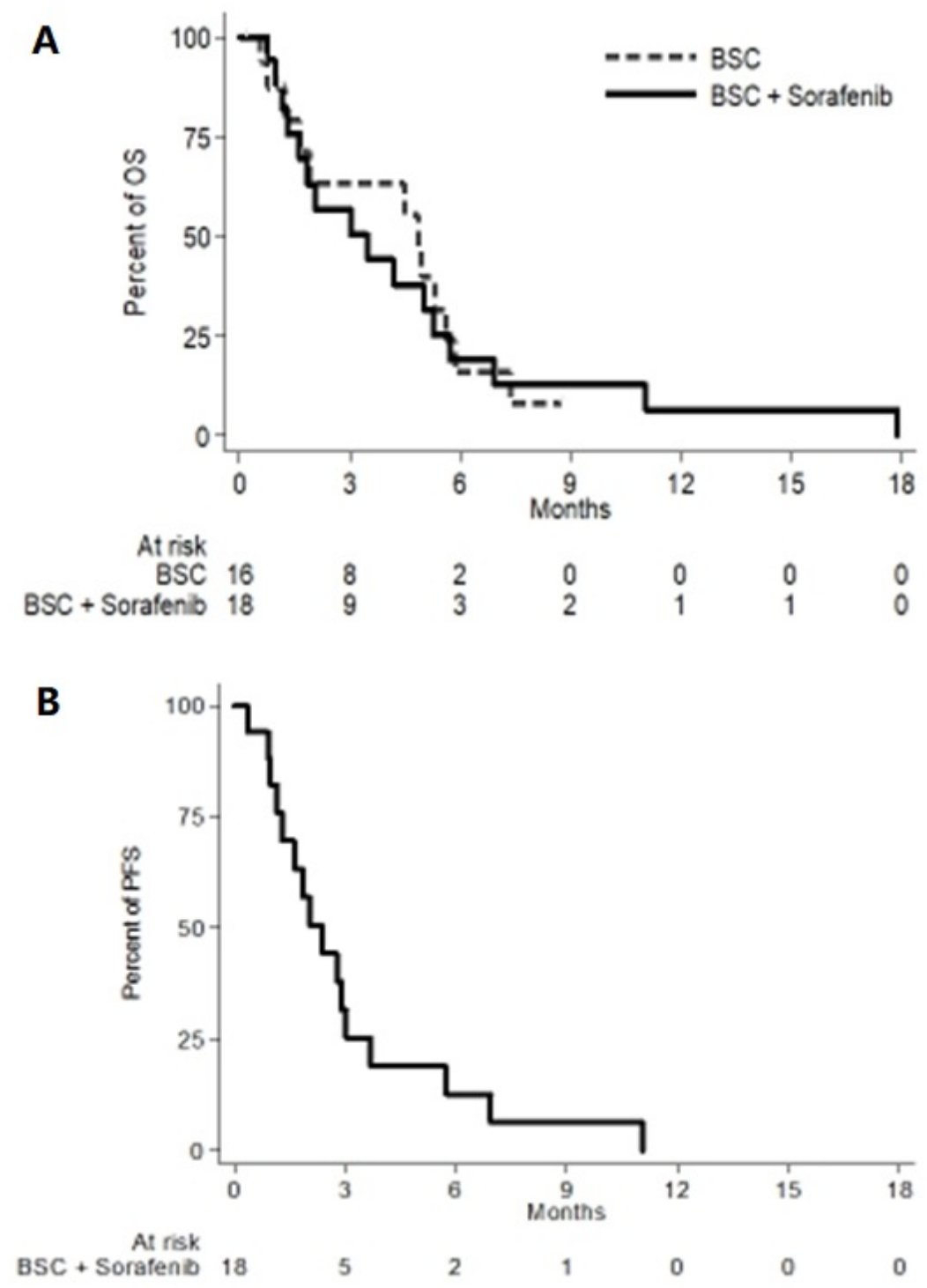

Figure 4. Overall survival (A) and progression-free survival (B) curves.

of the impact of treatment on QoL.

\section{DISCUSSION}

BOOST failed in its enrolment target. This was surprising for us, because the study was born within a clinical group that in the past had been extremely effective in conducting randomized clinical trials ${ }^{[16]}$. At the beginning of the study, the reason for the slow engagement of the investigators was surely the drug completely in charge of the local, enrolling institution. However, even later, when, thanks to the AIFA funding, the drug could be provided to local investigators free of charge, the enrollment did not proceed as expected. We believe that several reasons could have contributed to this. Above all, being sorafenib available in clinical practice since 2007, oncologists and hepatologists, thanks to clinical experience, might have reduced over time their uncertainty regarding the identification of candidate patients, excluding those with worse clinical conditions, or eventually intensifying supportive care to downstage Child-Pugh from B (score 7) to A, and attempting the treatment with sorafenib in patients with a strong motivation. In this context, 
several investigators could have lost their equipoise regarding the study question.

However, unfortunately, the study question remains unanswered.

The two registered trials, Sharp and Asia Pacific, enrolled only a few dozen Child-Pugh B patients (overall less than the BOOST study); therefore, in several countries, reimbursement of sorafenib is allowed only for Child-Pugh A patients ${ }^{[10]}$.

Retrospective, uncontrolled, and real life studies on the efficacy of sorafenib in HCC have been reported $^{[17-22]}$. The prospective observational phase 4 GIDEON trial was the largest one. It enrolled 3371 patients treated with sorafenib, including 666 Child-Pugh B and 74 Child-Pugh C cases. As expected, survival was longer for Child-Pugh A patients compared to Child-Pugh B ones (13.6 months vs. 5.2 months, respectively). Occurrence of serious adverse events $(60 \%$ vs. $36 \%)$ and the rate of subsequent treatment discontinuation (40\% vs. 29\%) were higher in Child-Pugh B than in Child-Pugh A patients ${ }^{[21]}$.

A prospective, observational, multicenter study (INSIGHT) enrolled 791 patients treated with sorafenib in daily practice conditions. In this trial, 182 (23\%) Child-Pugh B patients were included. The median OS was 17.6, 8.1, and 5.6 months for Child-Pugh A, B, and C patients, respectively. In this study, adverse events were less frequent among Child-Pugh B and C patients (50.8\% and 30.8\%, respectively) compared to ChildPugh A ones (72.4\%), possibly due to the shorter duration of treatment ${ }^{[22]}$.

In an Italian multicenter, open-label, phase 2 trial including Child-Pugh A (234 cases) and B patients (63 cases), median survival was shorter (3.8 months $v s .10 .0$ months) and the rate of adverse events was similar or lower for Child-Pugh B compared to Child-Pugh A patients ${ }^{[20]}$.

Overall, compliance and toxicity data reported in the small group of patients treated within the BOOST study are consistent with other findings reported in the literature in the same setting.

Finally, McNamara et al. ${ }^{[23]}$ published a metanalysis of studies including HCC patients (both Child-Pugh A and B) treated with sorafenib. The results regarding 1684 Child-Pugh B patients are similar to those of the above-mentioned studies, with a worse prognosis than Child-Pugh A patients, due to their poorer liver function, but no conclusions on the efficacy of sorafenib might be drawn in these patients.

Despite the limitations of the present study, due to failure in reaching the enrolment goal, BOOST represents one of the few randomized lines of evidence available on the use of first-line sorafenib in ChildPugh B HCC patients. Very recently, the results of the PRODIGE 21 phase 2 trial were published, suggesting a potential benefit of sorafenib in patients with HCC and Child-Pugh B liver cirrhosis with ALBI score $1 / 2^{[24]}$; however, this needs to be confirmed prospectively. Similar results have been shown with lenvatinibn ${ }^{[25]}$, cabozantinib ${ }^{[26]}$, and ramucirumab ${ }^{[27]}$. Overall, these data suggest that a good baseline liver function - as indicated by a lower ALBI score - is associated with a better outcome and a milder toxicity in patients with advanced HCC treated with the above drugs.

We could not calculate ALBI scores for our patients since albumin and bilirubin values were collected as categorical variables. Similar to what happened to our trial, Labeur et al. ${ }^{[28]}$ reported the premature termination of a study aimed at personalizing sorafenib therapy in patients with HCC and Child-Pugh B7-8 liver function, assessing also sorafenib pharmacokinetics. However, only 5 out of the 45 planned patients were actually recruited, over a period of almost three years. 
Notwithstanding the very low statistical power of BOOST that does not allow drawing any firm conclusions, the lack of any positive efficacy signal and the very poor compliance to sorafenib, together with all the available evidence mentioned above, led us to believe that there is no more room for programming further similar trials. Unfortunately, the lack of generalizability of study results to Child-Pugh B patients will also affect new drugs in HCC. Patients with CP-B were either completely excluded from registered trials of regorafenib ${ }^{[29]}$ and lenvatinib ${ }^{[30]}$ or allowed only if the Child score was 7 in the nivolumab CheckMate 040 study (with only 49 patients actually enrolled in the expansion cohort) ${ }^{[31]}$. The analysis of this latter group of patients indicated that, similar to sorafenib, nivolumab can be administered to some patients with ChildPugh B liver function. However, the efficacy of nivolumab in this setting remains to be demonstrated. A retrospective analysis of a subgroup of 73 patients randomized in the CELESTIAL trial (51 to cabozantinib and 22 to placebo) who progressed to Child-Pugh B class at Week 8 of treatment showed that, while the OS benefit of cabozantinib over placebo was maintained, some Grade $3 / 4$ adverse events (fatigue, ascites, AST elevation, and thrombocytopenia) were more common in the cabozantinib arm, while others (palmarplantar erythrodysesthesia and hypertension) were more frequent in the placebo $\operatorname{arm}^{[32]}$.

Thus, systemic treatment of advanced HCC patients with Child-Pugh B liver cirrhosis will remain an unmet need in the next years as well.

In conclusion, the BOOST trial failed and suggests that, when a wide registration of drugs (i.e., not fully supported by scientific evidence) has occurred, it may preclude advancing the knowledge of the actual efficacy of these drugs in selected populations. In fact, due to the availability of a drug in the general practice setting, neither will the investigators propose nor will the patients accept to be randomized to BSC. This scenario, as well as the BOOST results, should inspire researchers and the industry to plan clinical trials in definitive populations early in the development plan and the regulators to ask for more definitive evidence before clearing drugs for the general use in practice ${ }^{[33]}$.

\section{DECLARATIONS}

\section{Acknowledgments}

We thank Giuliana Canzanella, Marilena Martino, Maria Teresa Ribecco, Fiorella Romano, Giovanni de Matteis, Alfonso Savio, Lucia Sparavigna, Antonia Del Giudice, Simona Bevilacqua, Manuela Florio, Valentina Barbato, Anna Gimigliano for trial management at the Clinical Trial Unit of the Istituto Nazionale Tumori, IRCCS, Fondazione G.Pascale, Napoli.

\section{Authors' contributions}

Contributed conception, design and implementation of the research, to the analysis and interpretation of the data and to the writing of the manuscript: Daniele G, Schettino C, Piccirillo MC, Gargiulo P, Perrone F, Gallo C, Arenare L, Daniele B

Contributed to acquisition and interpretation of data: Farinati F, Federico P, Tamberi S, Crivellari G, Barni S, Tortora R, Izzo F, Frassoldati A, Cavanna L, Mucciarini C, Bolondi L, Dinota A, Pelizzaro F, Di Maio M, Bilancia D

All the Authors give final approval of the manuscript to be submitted.

\section{Availability of data and materials}

Data of this study will be shared with publication upon reasonable and motivated request to the Principal Investigator of the study (bruno.daniele@aslnapoli1centro.it). The following IPD will be available for sharing: baseline characteristics of patients, treatment data, safety data, follow-up data. There will be no time limit for data sharing. 


\section{Financial support and sponsorship}

The study was supported by the Italian Drug Agency (AIFA) in May 2011 (code FARM84SA2X) for trial coordination activities.

\section{Conflict of interest}

Massimo Di Maio reports personal honoraria (for participation in meetings, advisory boards or for acting as consultant) from Bristol Myers Squibb, Merck Sharp \& Dohme, Pfizer, AstraZeneca, Eisai, Takeda, Janssen, Astellas, and institutional research grant from Tesaro.

Antonio Frassoldati reports personal honoraria for advisory board from Novartis and Roche, invited lectures Novartis, Pfizer, Lilly, AstraZeneca, Celgene.

Sandro Barni reports personal honoraria from Genomic Health, Bayer AG, Eli-Lilly, Roche, Italfarmaco, Eisai, Astellas, Mylan, Leo Pharma, Pharmanutra, Medac Pharma, Kyowa Kirin.

Luigi Cavanna reports travel/Accomodations/meeting expenses from Pfizer, Ipsen, Celgene and personal honoraria for consultancy from AstraZeneca, Merck.

Raffaella Tortora reports personal honoraria for advisory boards from Bayer and Abbvie.

Gennaro Daniele: reports personal honoraria for advisory board from Beigene, and travel support from Roche.

Luigi Bolondi reports personal honoraria from Bayer, BMS, Sirtex, Guerbet, Alfa Sigma.

Francesco Perrone reports grants, personal fee and non-financial support from Bayer, grants and personal fees from Incyte and Astra Zeneca, personal fees from Celgene, Sandoz, Pierre Fabre, Janssen Cilag, Roche, and grants from Pfizer.

Maria Carmela Piccirillo reports personal fees from Daichii Sankyo, GSK, MSD, grants from Roche, grants and personal fees from AstraZeneca, non-financial support from Bayer.

Bruno Daniele reports personal fees from Bayer, Eli Lilly, Ipsen, Astrazeneca, MSD; Sanofi Aventis, Roche, personal fees and payment for lectures from Eisai.

The other authors declared that there are no conflicts of interest.

\section{Ethical approval and consent to participate}

The study was promoted by the Istituto Nazionale per lo Studio e la Cura dei Tumori, IRCCS, Fondazione G. Pascale, Napoli, Italy. The study was performed in accordance with the Declaration of Helsinki. Ethics Committees at each participating Institution approved the study, and all the patients signed the informed consent before any study related procedure.

\section{Consent for publication}

Not applicable.

\section{Copyright}

(C) The Author(s) 2021.

\section{REFERENCES}

1. Cancer today - IARC database. Available from: https://gco.iarc.fr [Last accessed on 2 Aug 2021].

2. Siegel RL, Miller KD, Jemal A. Cancer statistics, 2019. CA A Cancer J Clin 2018;69:7-34. DOI PubMed

3. new prognostic system for hepatocellular carcinoma: a retrospective study of 435 patients: the Cancer of the Liver Italian Program (CLIP) investigators. Hepatology 1998;28:751-5. DOI PubMed

4. Llovet JM, Brú C, Bruix J. Prognosis of hepatocellular carcinoma: the BCLC staging classification. Semin Liver Dis 1999;19:329-38. DOI PubMed

5. validation of the CLIP score: a new prognostic system for patients with cirrhosis and hepatocellular carcinoma. The Cancer of the Liver Italian Program (CLIP) Investigators. Hepatology 2000;31:840-5. DOI PubMed

6. Lu LC, Shao YY, Chan SY, Hsu CH, Cheng AL. Clinical characteristics of advanced hepatocellular carcinoma patients with prolonged survival in the era of anti-angiogenic targeted-therapy. Anticancer Res 2014;34:1047-52. PubMed

7. Wilhelm SM, Carter C, Tang L, et al. BAY 43-9006 exhibits broad spectrum oral antitumor activity and targets the RAF/MEK/ERK pathway and receptor tyrosine kinases involved in tumor progression and angiogenesis. Cancer Res 2004;64:7099-109. DOI PubMed 
8. Llovet JM, Ricci S, Mazzaferro V, et al; SHARP Investigators Study Group. Sorafenib in advanced hepatocellular carcinoma. $N$ Engl $J$ Med 2008;359:378-90. DOI PubMed

9. Cheng A, Kang Y, Chen Z, et al. Efficacy and safety of sorafenib in patients in the Asia-Pacific region with advanced hepatocellular carcinoma: a phase III randomised, double-blind, placebo-controlled trial. Lancet Oncol 2009;10:25-34. DOI PubMed

10. Granito A, Bolondi L. Non-transplant therapies for patients with hepatocellular carcinoma and Child-Pugh-Turcotte class B cirrhosis. Lancet Oncol 2017;18:e101-12. DOI PubMed

11. Bruix J, Sherman M; Practice Guidelines Committee; American Association for the Study of Liver Diseases. Management of hepatocellular carcinoma. Hepatology 2005;42:1208-36. DOI

12. Aaronson NK, Ahmedzai S, Bergman B, et al. The European Organization for Research and Treatment of Cancer QLQ-C30: a qualityof-life instrument for use in international clinical trials in oncology. J Natl Cancer Inst 1993;85:365-76. DOI PubMed

13. Blazeby JM, Currie E, Zee BC, Chie WC, Poon RT, Garden OJ; EORTC Quality of Life Group. Development of a questionnaire module to supplement the EORTC QLQ-C30 to assess quality of life in patients with hepatocellular carcinoma, the EORTC QLQHCC18. Eur J Cancer 2004;40:2439-44. DOI PubMed

14. Eisenhauer EA, Therasse P, Bogaerts J, et al. New response evaluation criteria in solid tumours: revised RECIST guideline (version 1.1). Eur J Cancer 2009;45:228-47. DOI PubMed

15. Schemper M, Smith TL. A note on quantifying follow-up in studies of failure time. Control Clin Trials 1996;17:343-6. DOI PubMed

16. Perrone F, Gallo C, Daniele B, et al; Cancer of Liver Italian Program (CLIP) Investigators. Tamoxifen in the treatment of hepatocellular carcinoma: 5-year results of the CLIP-1 multicentre randomised controlled trial. Curr Pharm Des 2002;8:1013-9. DOI PubMed

17. Pinter M, Sieghart W, Graziadei I, et al. Sorafenib in unresectable hepatocellular carcinoma from mild to advanced stage liver cirrhosis. Oncologist 2009;14:70-6. DOI PubMed

18. Abou-Alfa GK, Amadori D, Santoro A, et al. Safety and efficacy of sorafenib in patients with hepatocellular carcinoma (HCC) and Child-Pugh A versus B cirrhosis. Gastrointest Cancer Res 2011;4:40-4. PubMed PMC

19. Kim JE, Ryoo BY, Ryu MH, et al. Sorafenib for hepatocellular carcinoma according to Child-Pugh class of liver function. Cancer Chemother Pharmacol 2011;68:1285-90. DOI PubMed

20. Pressiani T, Boni C, Rimassa L, et al. Sorafenib in patients with Child-Pugh class A and B advanced hepatocellular carcinoma: a prospective feasibility analysis. Ann Oncol 2013;24:406-11. DOI PubMed

21. Marrero JA, Kudo M, Venook AP, et al. Observational registry of sorafenib use in clinical practice across Child-Pugh subgroups: the GIDEON study. J Hepatol 2016;65:1140-7. DOI PubMed

22. Ganten TM, Stauber RE, Schott E, et al. Sorafenib in patients with hepatocellular carcinoma-results of the observational INSIGHT study. Clin Cancer Res 2017;23:5720-8. DOI PubMed

23. McNamara MG, Slagter AE, Nuttall C, et al. Sorafenib as first-line therapy in patients with advanced Child-Pugh B hepatocellular carcinoma-a meta-analysis. Eur J Cancer 2018;105:1-9. DOI PubMed

24. Blanc JF, Khemissa F, Bronowicki JP, et al; PRODIGE 21 collaborators. Phase 2 trial comparing sorafenib, pravastatin, their combination or supportive care in HCC with Child-Pugh B cirrhosis. Hepatol Int 2021;15:93-104. DOI PubMed

25. Vogel A, Frenette C, Sung MW, et al. Baseline liver function and outcomes in the phase III REFLECT study in patients with unresectable hepatocellular carcinoma (uHCC). J Clin Oncol 2020;38:524. DOI

26. Chan S, Miksad R, Cicin I, et al. Outcomes based on albumin-bilirubin (ALBI) grade in the phase III CELESTIAL trial of cabozantinib versus placebo in patients with advanced hepatocellular carcinoma (HCC). Ann Oncol 2019;30:ix45-6. DOI

27. Kudo M, Galle PR, Brandi G, et al. Effect of ramucirumab on ALBI grade in patients with advanced HCC: results from REACH and REACH-2. JHEP Rep 2021;3:100215. DOI PubMed PMC

28. Labeur TA, Achterbergh R, Takkenberg B, Van Delden O, Mathôt R, Klümpen HJ. Sorafenib for patients with hepatocellular carcinoma and Child-Pugh B liver cirrhosis: lessons learned from a terminated study. Oncologist 2020;25:e1274-9. DOI PubMed PMC

29. Bruix J, Qin S, Merle P, et al. Regorafenib for patients with hepatocellular carcinoma who progressed on sorafenib treatment (RESORCE): a randomised, double-blind, placebo-controlled, phase 3 trial. Lancet 2017;389:56-66. DOI PubMed

30. Kudo M, Finn RS, Qin S, et al. Lenvatinib versus sorafenib in first-line treatment of patients with unresectable hepatocellular carcinoma: a randomised phase 3 non-inferiority trial. Lancet 2018;391:1163-73. DOI PubMed

31. Kudo M, Matilla A, Santoro A, et al. CheckMate 040 cohort 5: A phase I/II study of nivolumab in patients with advanced hepatocellular carcinoma and Child-Pugh B cirrhosis. J Hepatol 2021;S0168-8278(21)00313. DOI PubMed

32. El-Khoueiry AB, Hanna DL, Llovet J, Kelley RK. Cabozantinib: an evolving therapy for hepatocellular carcinoma. Cancer Treat Rev 2021;98:102221. DOI PubMed

33. Daniele G, Costa N, Lorusso V, Costa-Maia J, Pache I, Pirisi M. Methodological assessment of HCC literature. Ann Oncol 2013;24 Suppl 2:ii6-14. DOI PubMed PMC 